\title{
The association of serum beta-2-microglobulin with autoantibody production and disease activity in patients with primary Sjögren's syndrome
}

\author{
(1) Duygu Tecer ${ }^{1}$, (1) Dilek Büyükşireci², (1) Zafer Günendi ${ }^{3}$, (1) Feride Göğüş ${ }^{3}$ \\ 1 University of Health Sciences Turkey, Gülhane Training and Research Hospital, Clinic of Rheumatology, Ankara, Turkey \\ 2 Hitit University, Erol Olcok Training and Research Hospital, Clinic of Physical Medicine and Rehabilitation, Corum, Turkey \\ ${ }^{3}$ Gazi University Faculty of Medicine, Department of Physical Medicine and Rehabilitation, Division of Rheumatology, Ankara, Turkey
}

\section{Date submitted:}

29.03.2020

Date accepted:

04.07.2020

Online publication date:

15.12.2020

\section{Corresponding Author:}

Duygu Tecer MD, University of Health Sciences Turkey, Gülhane Training and Research Hospital, Clinic of Rheumatology, Ankara, Turkey duygu-tecer@hotmail.com

ORCID:

orcid.org/0000-0002-8816-6181

Keywords: Primary Sjögren's syndrome, beta-2-microglobulin, ESSDAI, ESSPRI, biomarkers

\begin{abstract}
Aims: The aim of the this study is to evaluate beta-2-microglobulin (B2M) levels in patients with primary Sjögren's syndrome (pSS) and to investigate their correlation with serum biomarkers and disease activity indexes commonly used in daily clinical practice.
\end{abstract}

Methods: Eighty-one patients with pSS were included in this retrospective and cross-sectional study. Demographic data, clinical characteristics, B2M, immunoglobulin (Ig) A, IgG, IgM, erythrocyte sedimentation rate (ESR), C-reactive protein (CRP), serum complement (C) 3 and C4 levels, anti-nuclear antibody, rheumatoid factor (RF), anti-SSA and anti-SSB antibodies were obtained from the medical records. Disease activity was evaluated by the European League Against Rheumatism (EULAR) SS Disease Activity Index (ESSDAI) and EULAR SS Patients Reported Index (ESSPRI).

Results: Serum B2M level was significantly higher in patients with anti-SSA and anti-SSB antibody (median: $2.64 \mathrm{mg} / \mathrm{dL}$, minimum: $2.02 \mathrm{mg} / \mathrm{dL}$, maximum: $10.10 \mathrm{mg} / \mathrm{dL}$ ) than in only anti-SSA positive patients (median: $2.31 \mathrm{mg} / \mathrm{dL}$, minimum: $1.33 \mathrm{mg} / \mathrm{dL}$, maximum: $4.26 \mathrm{mg} /$ $\mathrm{dL}, \mathrm{p}=0.010$ ) and both antibody negative patients (median: $1.80 \mathrm{mg} / \mathrm{dL}$, minimum: $1.20 \mathrm{mg} /$ $\mathrm{dL}$, maximum: $2.65 \mathrm{mg} / \mathrm{dL}, \mathrm{p}<0.001)$. Also, patients with anti-SSA antibody have significantly higher serum B2M levels than anti-SSA and anti-SSB antibodies negative patients $(p=0.009)$. Serum B2M level was significantly correlated with ESSDAI $(r=0.482, p=0.001)$, serum lgG level $(r=0.374, p=0.001)$, IgA level $(r=0.341, p=0.002)$, RF levels $(r=0.412, p=0.021)$ and ESR $(r=0.239$, $\mathrm{p}: 0.031)$. There was no correlation between ESSPRI, IgM, CRP and serum B2M levels. ESSDAI was not correlated with C3 $(r=0.044, p=0.697), C 4(r=-0.053, p=0.640), E S R(r=0.111, p=0.326), C R P$ $(r=0.111, p=0.324)$ and $\lg G(r=0.154, p=0.169)$.

Conclusions: Although serum B2M levels were higher in autoantibody positive patients, it has a weak correlation with clinical disease activity.

\section{Introduction}

Sjögrens's syndrome (SS) is a chronic disease at the junction of systemic autoimmune disorders and lymphoproliferative conditions (1). SS is characterized by a chronic lymphocytic infiltration of exocrine glands, predominantly the salivary and lacrimal glands (2). The clinical spectrum of SS extends from dryness of the mucosal surfaces such as mouth and eyes to severe life-threatening condition such as vasculitis and lymphoma (3). Due to the systemic nature of the disease, almost every organ can be affected (4). Early detection of highrisk patients will reduce mortality and morbidity (5). Biomarkers offering an early diagnosis, predicting the disease outcome and therapy response are still a need that has not been met.

Beta-2-microglobulin (B2M) is a low-molecular weight protein with sequence homology to immunoglobulins (lg) and expressed on the surface of all nucleated cell. B2M is non-covalently linked to the alpha chain of major histocompatibility complex-class 1 and plays a role in the antigen presentation to cytotoxic (CD8+) 
T lymphocytes (6). Also, B2M complexes with a cluster of differentiation 1 (CD1), MR1, human leukocyte antigen (HLA)-E, HLA-F, HLA-G, neonatal FC receptor (FcRn) and human hemochromatosis protein (HFE)/HLA-H, which are related to mucosal immunity, tumor surveillance, maternofetal immune tolerance, iron metabolism, homeostasis of immunoglobulin (Ig) and albumin (7). During normal cell turn over, it is released into the body fluids and presents as a soluble form at a constant rate. Serum level of B2M has been established as a prognostic marker in solid organ malignancies, hematologic disorders, and various autoimmune diseases such as Crohn's disease, SS, systemic lupus erythematosus and rheumatoid arthritis (8-10).

Serum B2M is an independent predictor of the primary SS (pSS) development in subject with sicca symptoms (11). It has also been associated with extraglandular systemic involvement of pSS overall, organ specific manifestation of pSS and lymphoma development (12-19). However, to date, studies which evaluate the relationship between B2M and disease activity in patients with pSS are limited (20-22).

The aim of this study is to evaluate B2M levels in patients with pSS and to investigate their correlation with serum biomarkers and disease activity indexes commonly used in daily clinical practice to evaluate disease status.

\section{Methods}

This study was planned as a retrospective and cross-sectional study. Medical records of pSS patients who applied to our outpatient clinic between July 2016 and July 2017 were retrospectively scanned from the electronic database of our tertiary hospital. In our rheumatology department, standard clinical and laboratory investigation are made at each visit to assess the disease activity and organ involvement of patients with pSS. After taking accurate medical history and carefully performing physical examination, erythrocyte sedimentation rate (ESR), C-reactive protein (CRP), $\mathrm{B} 2 \mathrm{M}$, IgA, IgG, IgM, serum complement (C) 3 and C4 levels are routinely evaluated as the predictors of disease activity. Disease activity is assessed with European League Against Rheumatism (EULAR) SS Disease Activity Index (ESSDAI) and EULAR SS Patients Reported Index (ESSPRI). In the present study, clinical and laboratory values of the most recent visit were evaluated. Patients with impaired renal function, concomitant autoimmune disease, known solid organ malignancies, or lymphoproliferative disorders were excluded, as these conditions are associated with increased serum B2M levels. Consequently, a total of 81 pSS patients who fulfilled 2002 American European Consensus Group criteria for diagnosing pSS were enrolled (23). The study was approved by the Committee on Human Research Ethics at Zekai Tahir Burak Women's Health Training and Research Hospital (dated: 28.02.1017, decision number: 39/2017).

Demographic data, clinical characteristics and laboratory results, including anti-nuclear antibody (ANA), rheumatoid factor
(RF), anti-SSA and anti-SSB antibodies, ESR, CRP, serum levels of $\mathrm{B} 2 \mathrm{M}, \lg \mathrm{A}, \lg \mathrm{G}, \lg \mathrm{M}, \mathrm{C} 3$ and $\mathrm{C}$ were obtained from electronic medical records.

In our hospital, serum concentrations of $\lg A, \lg G$, and $\operatorname{lgM}$, as well as serum levels of $\mathrm{C} 3$ and $\mathrm{C} 4$, were measured by laser nephelometry. Normal values were 79 to $152 \mathrm{mg} / \mathrm{dL}$ for C3 and 16 to $38 \mathrm{~g} / \mathrm{L}$ for C4. ANA were detected by indirect immunofluorescence HEp-2 cells. Anti-SSA and anti-SSB antibody levels were determined by commercial enzyme linked immunosorbent assay. The serum B2M level was determined using nephelometry. According to the recommendations of manufacturer, a serum B2M value of $1.8 \mathrm{mg} / \mathrm{dL}$ or more was considered to be increased.

The ESSPRI is a patient-administered questionnaire which evaluates symptoms including pain, fatigue and dryness. Each individual symptom is evaluated with an eleven-point numerical scale. The scale is composed of 0 (no symptoms) to 10 (severe symptoms) scores. The final ESSPRI score is calculated by taking the average scores of these domains. Total ESSPRI score ranges from 0 to 10 . The ESSPRI scores of $<5$ define low disease activity and scores of $\geq 5$ define high disease activity $(24,25)$.

The ESSDAI is a physician-based index for the assessment of the disease activity in patients with pSS. This index includes 12 domains as follows: constitutional, lymphadenopathy, glandular, articular, cutaneous, respiratory, renal, muscular, peripheral nervous system, central nervous system, hematological and biological. Each domain is scored from 0 to 3 or 4 according to activity levels. Total ESSDAI score is calculated by taking the sum scores of these domains score with a maximum severity score of 123 . ESSDAI score $<5$ is defined as low disease activity, $5 \leq$ ESSDAI score $\leq 13$ is defined as moderate disease activity, and ESSDAI score $\geq 14$ is defined as high disease activity $(25,26)$.

\section{Statistical Analysis}

All data were analyzed using the Statistical Package for Social Sciences (SPSS Inc., Chicago, IL, USA) 16.0 program for Windows. The variables were investigated using visual and analytical methods to determine whether they were normally distributed. Continuous values were expressed as mean \pm standard deviation and categorical variables as number and percentage. Among serum Ig, C3, C4 and B2M levels were not normally distributed and the Kruskal-Wallis test was conducted to compare these parameters among the antibody status. The Mann-Whitney $U$ test was performed to test the significance of pairwise differences using Bonferroni correction to adjust for multiple comparisons. Spearman's correlation coefficient was used to evaluate the linear relationship between the predictive variables. A value of $p<0.05$ was considered to be statistically significant. 


\section{Results}

Clinical and immunologic features of patients are presented in Table 1. C3 and C4 levels were decreased in 4 (4.9\%) and 7 (8.6\%) patients, respectively. Patients with anti-SSA antibody or both anti-SSA and anti-SSB antibody had statistically significant higher serum B2M levels, IgG and IgA than those without autoantibodies (Table 2). The serum B2M level had a weak correlation with ESSDAI $(r=0.482, p=0.001)$, serum IgG level $(r=0.374, p=0.001)$, serum IgA level $(r=0.341, p=0.002)$, serum RF levels $(r=0.412, p=0.021)$ and ESR $(r=0.239$, $p=0.031)$. Serum B2M levels were not correlated with ESSPRI $(r=0.089, p=0.429), \lg M(r=0.009, p=0.934), C R P(r=-0.105$, $\mathrm{p}=0.352$ ).

According to the ESSPRI score, $47(58 \%)$ patients had low disease activity. Although patients with high disease activity tended to have higher serum B2M levels, there was no statistically significant difference $(2.13 \pm 0.69,2.48 \pm 1.62$ respectively, $p=0.531$ ).

According to the ESSDAI score, 38 (46.9\%) of 71 patients had low disease activity, $31(38.3 \%)$ patients had moderate disease activity and $12(14.8 \%)$ patients had high disease activity. The median (minimum-maximum) serum B2M level was $1.83(1.20-2.59)$ in patients with low disease activity, 2.46 (1.55-5.41) in those with moderate disease activity, and 2.97 (1.60-10.20) in those with high disease activity. Patients with low disease activity had significantly lower serum B2M levels than patients with moderate disease activity $(p<0.001)$ and high disease activity $(p=0.001)$. Although patients with high disease activity tended to have higher serum B2M levels, there was no statistically significant difference between high and moderate disease activities $(p=0.147)$. Also, ESSDAl score was not correlated with C3 $(r=0.044, p=0.697), C 4(r=-0.053, p=0.640)$, ESR ( $r=0.111, p=0.326), \operatorname{CRP}(r=0.111, p=0.324)$, and $\lg$ $(r=0.154, p=0.169)$.

\section{Discussion}

In this study which investigated the usefulness of serum B2M level in evaluating disease activity of pSS patients, it was found that serum B2M level was positively correlated with ESSDAl, serum IgG level, IgA level, RF level and ESR. In addition, serum B2M level was significantly higher in patients with anti-SSA antibody or both anti-SSA and anti-SSB antibody than in those without autoantibodies. Furthermore, patients with anti-SSA and anti-SSB antibody had significantly higher serum B2M levels than patients with only anti-SSA antibody. In another study, Gottenberg et al. (19) evaluated the association between autoantibody production and serum B2M levels in patients with pSS. Similar to our study, they reported that the serum B2M level was significantly higher in patients with anti-SSA and anti-SSB antibodies than in those with anti-SSA antibody alone or those without anti-SSA and anti-SSB autoantibodies. In addition, they showed that serum B2M level was significantly correlated with serum RF ( $r=0.33, p=0.001)$, IgG $(r=0.42, p=0.001)$, and $E S R$ $(r=0.39, p=0.001)(19)$.

During normal cell turn over, B2M is primarily released from immune-related cells including macrophages, active $T$ and $B$ lymphocytes in to most biological fluids (7). Following glomerular filtration, B2M is completely reabsorbed and catabolized in the

\begin{tabular}{ll}
\hline Table 1. Clinical and immunological features of patients with primary Sjögren's syndrome \\
\hline Age, years & $51.19 \pm 10.83$ \\
\hline Male/female & $2 / 79$ \\
\hline Rheumatoid factor positive $(\mathrm{n})(\%)$ & $28(34.6 \%)$ \\
\hline Serum IgA level (mg/dL) & $251.00(75.30-637.00)$ \\
\hline Serum IgG level (mg/dL) & $1270.00(773.00-2970.00)$ \\
\hline Serum IgM level (mg/dL) & $113.00(44.50-269.00)$ \\
\hline Serum C3 level (mg/dL) & $125.00(52.50-186.00)$ \\
\hline Serum C4 level (mg/dL) & $23.30(7.38-46.30)$ \\
\hline CRP mg/L & $3.66(1.04-17.30)$ \\
\hline ESR (mm/h) & $19.00(2.00-66.00)$ \\
\hline Anti-SSA and anti-SSB antibodies negative & $40(49.4 \%)$ \\
\hline Only anti-SSA antibody positive & $22(27.2 \%)$ \\
\hline Anti-SSA and anti-SSB antibodies positive & $19(23.5 \%)$ \\
\hline Serum beta-2-microglobulin (mg/dL) & $2.02(1.20-10.20)$ \\
\hline ESSDAl & $5.00(0-26)$ \\
\hline ESSPRI & $4.00(0.00-9.33)$ \\
\hline Data shown as median (minimum-maximum) where otherwise stated. & \\
\hline Ig: Immunoglobulin, CRP: C-reactive protein, ESR: Erythrocyte sedimentation rate, EULAR: European League Against Rheumatism, ESSDAl: EULAR Sjögrens's \\
\hline Syndrome Disease Activity Index, ESSPRI: EULAR Sjögrens's Syndrome Patients Reported Index
\end{tabular}




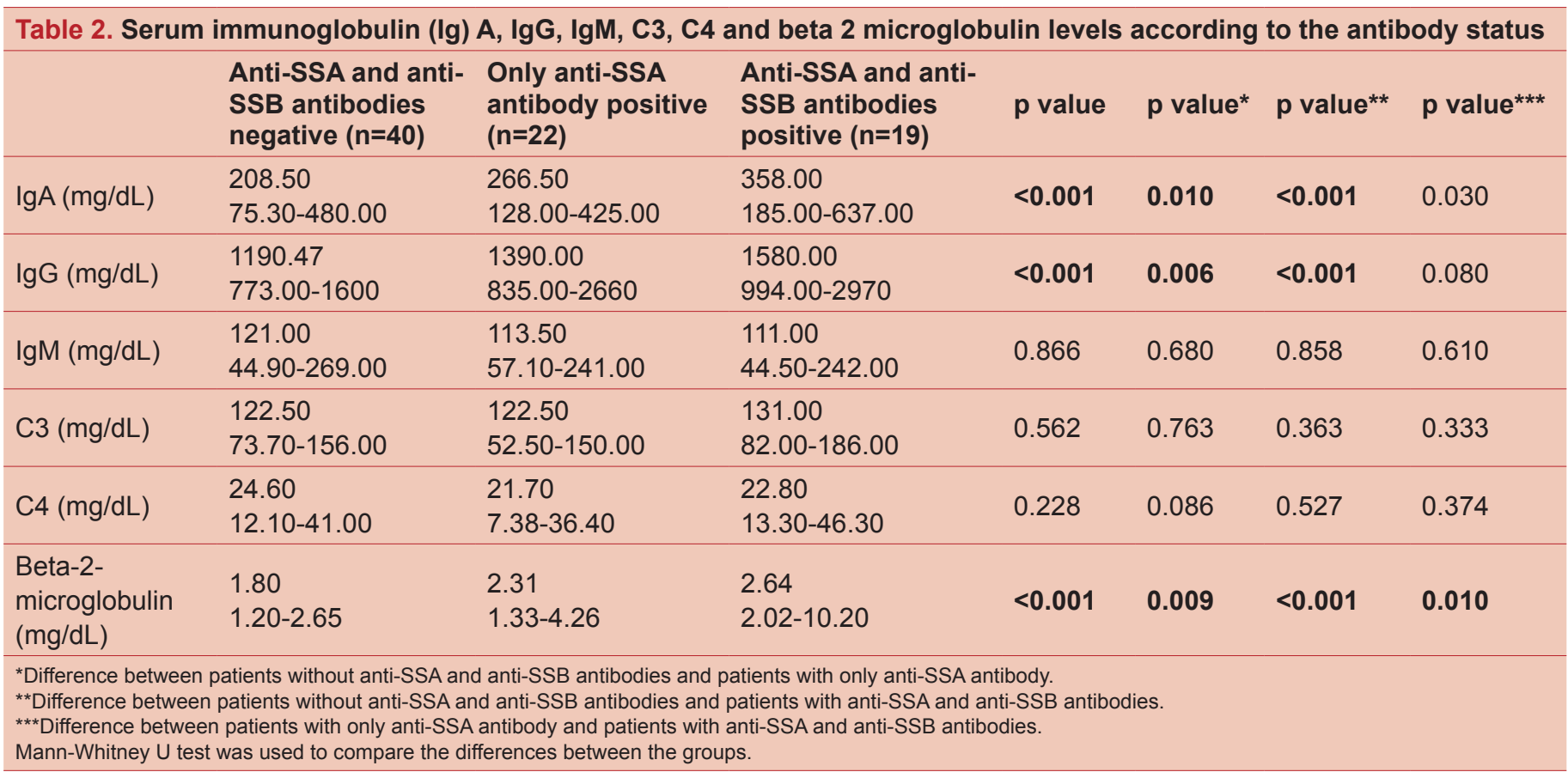

proximal renal tubules (27). The abnormality of serum or urine $\mathrm{B} 2 \mathrm{M}$ level is associated with various hematologic malignancies, autoimmune disease, and renal disorders $(7,28,29)$.

Although the specific function of serum B2M remains unknown in pSS, previous studies reported that B2M level was increased in pSS patients with particular individual clinical manifestations. Hatron et al. (17) reported that B2M was increased in the serum of pSS patients with latent alveolitis. Lahdensuo and Korpela (18) reported that pSS patients with pulmonary hyperinflation had elevated serum B2M levels and disturbed lung function was correlated with high serum B2M levels. Pertovaara et al. (13) showed that high level of serum B2M was one of the best predictors of the development of distal renal tubular acidosis in pSS patients. In addition, a longitudinal cohort study contucted by Pertovaara et al. (14) showed that baseline serum B2M level was a significant predictor of lymphoma development in pSS patients (Odds ratio: 1.9; 95\% confidence interval: 1.1 to 3.4 ; $\mathrm{p}=0.031)$.

In our pSS patient, ESSDAI score was correlated significantly with serum B2M levels $(r=0.482, p=0.001)$ but was not correlated with serum levels of $\lg G(r=0.154, p=0.169)$ and C4 $(r=-0.053, p=0.640)$, although these parameters are included in the ESSDAI. Like our study, Pertovaara and Korpela (20) reported that serum B2M level was significantly correlated with the ESSDAI score $(r=0.383, p=0.001)$ and not correlated with serum levels of IgG $(r=0.014, p=0.906)$ or C4 $(r=-0.105$, $p=0.359$ ). Authors suggested that this was presumably due to the fact that serum levels of $\operatorname{lgG}$ and $\mathrm{C} 4$ were given a rather low weight in the calculation of ESSDAI score. In another study conducted by James et al. (30), it was reported that serum B2M level was an independent predictor of ESSDAI scores
(30). Unlike our study, in their study, according to the Poisson regression of serum $\mathrm{B} 2 \mathrm{M}$ level against the ESDDAI domain, serum B2M level was significantly associated with biological domains of ESSDAI. Also, Gottenberg et al. (21) evaluated the baseline clinical and immunological features of the Assessment of Systemic Signs and Evolution of SS cohort and showed that pSS patients with elevated serum B2M levels had higher ESSDAI scores at enrollment.

In our study, serum B2M level was not correlated with the ESSPRI score, which evaluates patient's dryness, fatigue and pain. Similar to our results, Gottenberg et al. (21) observed that ESSPRI was not correlated with serum B2M levels. This is probably due to the fact that ESSPRI is based on the subjective perception of patients.

Several limitations to the present study warrant attention. The small sample size is a major limitation of our study. Larger sample size may be needed to identify serum biomarkers in systemic autoimmune diseases, which are heterogeneous diseases. The cross-sectional design is another limitation of this study. This study does not provide sufficient results to investigate possible causality and effect relationship. Also, our results do not give information about the changes in serum B2M level with disease duration, disease activity and treatment response. Despite these limitations, our results support the previous findings that serum B2M is a considerable biomarker for assessing disease activity of pSS.

\section{Conclusion}

As a conclusion, serum B2M level may be a useful biomarker in evaluating disease activity of pSS. In the future, there is a need of well-designed, prospective, controlled studies with a 
larger sample size to validate clinical value of this simple, widely available, and inexpensive blood test as an activity marker in pSS patients.

\section{Ethics}

Ethics Committee Approval: The study was approved by the Committee on Human Research Ethics at Zekai Tahir Burak Women's Health Training and Research Hospital (dated: 28.02.1017, decision number: 39/2017).

Informed Consent: Retrospective study.

Peer-review: Externally peer-reviewed.

\section{Authorship Contributions}

Surgical and Medical Practices: D.T., D.B., Z.G., F.G., Concept: D.T., D.B., Z.G., F.G., Design: D.T., D.B., Z.G., F.G., Data Collection or Processing: D.T., D.B., Z.G., F.G., Analysis or Interpretation: D.T., D.B., Z.G., F.G., Literature Search: D.T., D.B., Z.G., F.G., Writing: D.T., D.B., Z.G., F.G.

Conflict of Interest: No conflict of interest was declared by the authors.

Financial Disclosure: The authors declared that this study received no financial support.

\section{References}

1. Ferro F, Marcucci E, Orlandi M, Baldini C, Bartoloni-Bocci E. One year in review 2017: primary Sjogren's syndrome. Clin Exp Rheumatol. 2017;35:179-191.

2. Fox RI. Sjogren's syndrome. Lancet. 2005;366:321-331.

3. Barone F, Colafrancesco S. Sjogren's syndrome: from pathogenesis to novel therapeutic targets. Clin Exp Rheumatol. 2016;34(Suppl 4):58-62.

4. Goules AV, Tzioufas AG. Primary Sjogren's syndrome: clinical phenotypes, outcome and the development of biomarkers. Immunol Res. 2017;65:331-344.

5. Brito-Zeron P, Theander E, Baldini C, et al. Early diagnosis of primary Sjogren's syndrome: EULAR-SS task force clinical recommendations. Expert Rev Clin Immunol. 2016;12:137-156.

6. Chiou SJ, Chen $\mathrm{CH}$. Decipher beta2-microglobulin: gainor loss-of-function (a mini-review). Med Sci Monit Basic Res. 2013;19:271-273.

7. Argyropoulos $\mathrm{CP}$, Chen SS, Ng YH, et al. Rediscovering beta-2 microglobulin as a biomarker across the spectrum of kidney diseases. Front Med (Lausanne). 2017;4:73.

8. Yeung $\mathrm{CK}$, Wong $\mathrm{KL}$, Wong WS, Chan $\mathrm{KH}$. Beta 2-microglobulin and systemic lupus erythematosus. J Rheumatol. 1986;13:1053-1058.

9. Yang J, Qian J, Wezeman M, et al. Targeting beta2microglobulin for induction of tumor apoptosis in human hematological malignancies. Cancer Cell. 2006;10:295307.
10. Yilmaz B, Koklu S, Yuksel O, Arslan S. Serum beta 2-microglobulin as a biomarker in inflammatory bowel disease. World J Gastroenterol. 2014;20:10916-10920.

11. Pertovaara $\mathrm{M}$, Korpela $\mathrm{M}$, Uusitalo $\mathrm{H}$, et al. Clinical follow up study of 87 patients with sicca symptoms (dryness of eyes or mouth, or both). Ann Rheum Dis. 1999;58:423-427.

12. Pertovaara M, Korpela M, Kouri T, Pasternack A. The occurrence of renal involvement in primary Sjogren's syndrome: A study of 78 patients. Rheumatology (Oxford). 1999;38:1113-1120.

13. Pertovaara $M$, Korpela $M$, Pasternack $A$. Factors predictive of renal involvement in patients with primary Sjogren's syndrome. Clin Nephrol. 2001;56:10-18.

14. Pertovaara M, Pukkala E, Laippala P, Miettinen A, Pasternack A. A longitudinal cohort study of Finnish patients with primary Sjogren's syndrome: clinical, immunological, and epidemiological aspects. Ann Rheum Dis. 2001;60:467-472.

15. Pertovaara M, Korpela M, Saarelainen S, et al. Longterm follow-up study of pulmonary findings in patients with primary Sjogren's syndrome. Scand J Rheumatol. 2004;33:343-348.

16. Michalski JP, Daniels TE, Talal N, Grey HM. Beta2 microglobulin and lymphocytic infiltration in Sjogren's syndrome. N Engl J Med. 1975;293:1228-1231.

17. Hatron PY, Wallaert B, Gosset D, et al. Subclinical lung inflammation in primary Sjogren's syndrome. Relationship between bronchoalveolar lavage cellular analysis findings and characteristics of the disease. Arthritis Rheum. 1987;30:1226-1231.

18. Lahdensuo A, Korpela M. Pulmonary findings in patients with primary Sjogren's syndrome. Chest. 1995;108:316-319.

19. Gottenberg JE, Busson M, Cohen-Solal J, et al. Correlation of serum B lymphocyte stimulator and beta2 microglobulin with autoantibody secretion and systemic involvement in primary Sjogren's syndrome. Ann Rheum Dis. 2005;64:1050-1055.

20. Pertovaara M, Korpela M. Serum beta2 microglobulin correlates with the new ESSDAI in patients with Sjogren's syndrome. Ann Rheum Dis. 2011;70:2236-2237.

21. Gottenberg JE, Seror R, Miceli-Richard C, et al. Serum levels of beta2-microglobulin and free light chains of immunoglobulins are associated with systemic disease activity in primary Sjogren's syndrome. Data at enrollment in the prospective ASSESS cohort. PLoS One. 2013;8:e59868.

22. Pertovaara $M$, Parkkila $S$, Korpela M. Anti-carbonic anhydrase autoantibodies and serum beta-2 microglobulin correlate with the ClinESSDAI score in patients with Sjogren's syndrome. Clin Exp Rheumatol. 2017;35:351.

23. Vitali C, Bombardieri S, Jonsson R, et al. Classification criteria for Sjogren's syndrome: a revised version of the European criteria proposed by the American-European Consensus Group. Ann Rheum Dis. 2002;61:554-558. 
24. Seror R, Ravaud P, Mariette X, et al. EULAR Sjogren's Syndrome Patient Reported Index (ESSPRI): development of a consensus patient index for primary Sjogren's syndrome. Ann Rheum Dis. 2011;70:968-972.

25. Seror $R$, Bootsma $H$, Saraux $A$, et al. Defining disease activity states and clinically meaningful improvement in primary Sjogren's syndrome with EULAR primary Sjogren's syndrome disease activity (ESSDAI) and patient-reported indexes (ESSPRI). Ann Rheum Dis. 2016;75:382-389.

26. Seror R, Ravaud P, Bowman SJ, et al. EULAR Sjogren's syndrome disease activity index: development of a consensus systemic disease activity index for primary Sjogren's syndrome. Ann Rheum Dis. 2010;69:1103-1109.

27. Li L, Dong M, Wang XG. The Implication and Significance of Beta 2 Microglobulin: A Conservative Multifunctional Regulator. Chin Med J (Engl). 2016;129:448-455.
28. Yoo C, Yoon DH, Suh C. Serum beta-2 microglobulin in malignant lymphomas: an old but powerful prognostic factor. Blood Res. 2014;49:148-153.

29. Choe JY, Park SH, Kim SK. Urine beta2-microglobulin is associated with clinical disease activity and renal involvement in female patients with systemic lupus erythematosus. Lupus. 2014;23:1486-1493.

30. James K, Chipeta C, Parker A, et al. B-cell activity markers are associated with different disease activity domains in primary Sjogren's syndrome. Rheumatology (Oxford). 2018;57:1222-1227. 